\title{
A influência do pré-natal, parto e intercorrências mamárias no tempo amamentação
}

The influence of prenatal care, childbirth and breast complications on breastfeeding-

lactation time

La influencia de la atención prenatal, el parto y las complicaciones mamarias en el tiempo

de lactancia-lactancia

Alice Gontijo Cançado ${ }^{1}$, Ana Carolina Pereira Maciel ${ }^{1}$, Fabiana Francia Abreu ${ }^{1}$, Fernanda Ferradeira Latorre1, Laura de Almeida Rosa ${ }^{2}$, Carolina Andrade Vitoi ${ }^{2}$, Patrícia de Oliveira Lima², Sirleide Corrêa Rangel ${ }^{2}$, Juliana Barroso Zimmermmann ${ }^{1,2,3 *}$.

\section{RESUMO}

Objetivo: Avaliar a frequência de amamentação de puérperas associando ao tipo de parto e verificar quais fatores são limitadores do período de lactação. Métodos: Foram entrevistadas puérperas atendidas pelo Serviço de Obstetrícia da Faculdade de Medicina de Barbacena e da Universidade Federal de Juiz de Fora, sendo essas submetidas à anamnese e questionadas sobre antecedentes, tipo de pré-natal, via de parto, intercorrências mamárias, regime de aleitamento e fatores interferentes. Resultados: Não houve associação estatística entre amamentação inicial e tipo de pré-natal, idade, número de gestações, partos e tipo de parto. Foi identificado hipogalactia como principal fator de interferência aos 15 dias de pós-parto, aos 21 foi agalactia, e aos 75 e 180 dias predominou a indicação do pediatra. Conclusão: A grande maioria das pacientes saem da maternidade amentando de forma exclusiva. Entretanto, com o passar dos dias há queda importante da lactação exclusiva, seja por hipogalactia ou agalactia. O curioso, nesse estudo, foi a orientação do pediatra que apareceu como causa de suspensão da lactação. A cesariana foi fator de risco para a lactação não exclusiva.

Palavras-chave: Amamentação, Parto, Cesárea, Lactação.

\begin{abstract}
Objective: Assessment of the frequency of breastfeeding of puerperal women associating with the type of delivery and checking which factors are limiting the lactation period. Methods: Postpartum women attended by the Obstetrics Service of the Faculty of Medicine of Barbacena and the Federal University of Juiz de Fora were interviewed, who were submitted to anamnesis and questioned about background, type of prenatal care, way of delivery, breast complications, breastfeeding interfering factors. Results: There was no statistical association between initial breastfeeding and type of prenatal care, age, number of pregnancies, deliveries and type of delivery. Hypogalactia was identified as the main interference factor at 15 days postpartum, at 21 it was agalactia, and at 75 and 180 days the pediatrician's indication predominated. Conclusion: The vast majority of patients leave the maternity hospital exclusively breastfeeding. However, as the days go by, there is an important drop in exclusive lactation, either due to hypogalactia or agalactia. The curious thing about this study was the guidance of the pediatrician, who appeared as a cause of suspension of lactation. Caesarean section was a risk factor for non-exclusive lactation.
\end{abstract}

Keywords: Breast-feeding, Childbirth, Caesarean, Lactation.

${ }_{1}^{1}$ Faculdade de Medicina de Barbacena (FAME), Barbacena - MG. *E-mail: julianabz@uol.com.br

${ }^{2}$ Hospital Universitário da Universidade Federal de Juiz de Fora (HU-UFJF), Juiz de Fora - MG.

3 Universidade Federal de Juiz de Fora (UFJF), Juiz de Fora - MG. 


\section{RESUMEN}

Objetivo: Evaluación de la frecuencia de la lactancia materna de las puérperas asociándola al tipo de parto y comprobando qué factores limitan el período de lactancia. Métodos: Se entrevistó a mujeres posparto atendidas por el Servicio de Obstetricia de la Facultad de Medicina de Barbacena y la Universidad Federal de Juiz de Fora, quienes fueron sometidas a anamnesis y cuestionadas sobre antecedentes, tipo de atención prenatal, forma de parto, complicaciones mamarias, factores interferentes de la lactancia materna. Resultados: No hubo asociación estadística entre la lactancia materna inicial y el tipo de atención prenatal, edad, número de embarazos, partos y tipo de parto. La hipogalaxia se identificó como el principal factor de interferencia a los 15 días posparto, a los 21 fue agalaxia, ya los 75 y 180 días predominó la indicación del pediatra. Conclusión: La gran mayoría de pacientes abandonan la maternidad con lactancia materna exclusiva. Sin embargo, a medida que pasan los días, se produce un descenso importante de la lactancia exclusiva, ya sea por hipogalactia o agalactia. Lo curioso de este estudio fue la orientación del pediatra, quien apareció como causa de suspensión de la lactancia. La cesárea fue un factor de riesgo para la lactancia no exclusiva

Palabras clave: Amamantamiento, Parto, Cesariano, Lactancia.

\section{INTRODUÇÃO}

O aleitamento materno é uma inteligente forma natural de vínculo, amor, cuidado e nutrição para o bebê, constituindo uma interessante medida para redução da morbimortalidade infantil (HORTON S, et al., 1996). Em 1990, a Organização Mundial da Saúde (OMS) e o Fundo das Nações Unidas para Infância (UNICEF) mobilizaram-se em prol da proteção e apoio à amamentação, criando a Iniciativa Hospital Amigo da Criança (IHAC), estabelecendo "Dez Passos para o Sucesso do Aleitamento Materno" (JELLIFFE DB, 1972; ROTH DE, et al., 2008).

O leite materno é um alimento completo e a amamentação promove vários benefícios tanto para a criança quanto para mãe. Dentre as vantagens para o recém-nascido tem-se melhor digestibilidade, evita cólica abdominal e diarréia, composição química balanceada, ausência de princípios alergênicos, proteção contra infecção e favorece o desenvolvimento neurológico e emocional do bebê (VICTORA GG, et al., 1987). Para a mãe, são relacionados ao baixo custo, ajuda no retorno da forma física, redução do risco de desenvolvimento de câncer de mama e ovário, impede a ovulação e reduz o risco de novas gestações, auxilia o útero a retornar ao seu tamanho normal e reduz a chance de anemia por sangramento pós-parto (ESCUDER MML, et al., 2003).

O aleitamento também interfere na vida futura do bebê. Sendo assim, pode-se dizer que crianças amamentadas no peito tem menos chances de apresentar diarréia e doenças pulmonares no primeiro ano de vida. Cita-se também a redução da obesidade na primeira infância (VICTORA GG, et al., 1987; ESCUDER MML, et al., 2003; VICTORA GG, et al., 1992; CESAR JA, et al., 1999). Segundo Siqueira RS e Monteiro CA (2007), a prevalência de obesidade entre crianças (6 a 14 anos) na cidade de São Paulo de uma escola particular de alto padrão econômico foi de $26 \%$. Esses autores identificaram que o risco de obesidade em crianças que nunca receberam aleitamento materno foi duas vezes superior $(O R=2,06$; IC 95\%: 1,02; 4,16) ao risco das demais crianças. Owen CG, et al. (2005), em metanálise, identificaram que o aleitamento materno estava associado a um menor risco de obesidade quando comparado ao aleitamento artificial $(\mathrm{RO}=0,87$; IC 95\%: 0,$85 ; 0,90)$.

Entretanto, existem diversos fatores que influenciam no aleitamento, como questões culturais, nível socioeconômico, grau de escolaridade, idade e trabalho materno, urbanização, condições de parto, incentivo do companheiro e familiares e o desejo atual da mulher em amamentar (WHO, 2001; MCVEA KL, et al., 2000). Por isso, o aleitamento materno pode ser considerado uma "jornada pessoal de imersão" precisando de compromisso materno, adaptação e apoio de diferentes fontes (BOCCOLINI CS, 2012; BRASIL, 2015; MARTINS TC, et al., 2019).

Baseado no exposto, o objetivo do estudo foi avaliar a frequência de amamentação de puérperas associando ao tipo de parto e verificar quais fatores são limitadores do período de lactação. 


\section{MÉTODOS}

Trata-se de uma coorte aberta onde foram estudadas lactantes atendidas pelo Serviço de Obstetrícia da Faculdade de Medicina de Barbacena e da Universidade Federal de Juiz de Fora. Todas foram submetidas à anamnese e pesquisa de antecedentes pessoais e familiares (idade, número de filhos, filhos de outros relacionamentos, doenças de base, tratamentos prévios, cirurgias mamárias, uso de medicação, tabagismo, alcoolismo), tipo de parto, tempo em trabalho de parto, apoio à amamentação pela família e marido. A seguir, foi realizada a avaliação das mamas. Na inspeção, observou-se a cor das mamas, mamilos, áreas de retração e abaulamento.

Após, as pacientes foram colocadas em decúbito dorsal, com a palpação de toda a área da mama, seguida pela expressão do mamilo para identificar drenagem papilar patológica. Foram incluídas todas as lactantes atendidas neste período e excluídas aquelas com prótese de silicone ou mamoplastia redutora. Os dois primeiros controles foram presenciais, com avaliação clínica. Após os dois primeiros controles, as pacientes foram contactadas por telefone para conhecermos o regime de amamentação. O terceiro controle está sendo realizado entre 75 a 140 dias do parto e o último controle após 140 dias do parto. $O$ gráfico abaixo identifica o número de pacientes abordadas em cada controle.

Observa-se que algumas pacientes ainda não responderam o terceiro e quarto controle. Em relação ao segundo controle ambulatorial, 6 pacientes deixaram de realizá-lo (Figura 1).

Figura 1 - Número de pacientes puérperas na abordagem de cada controle - hospitalar ou ambulatorial.

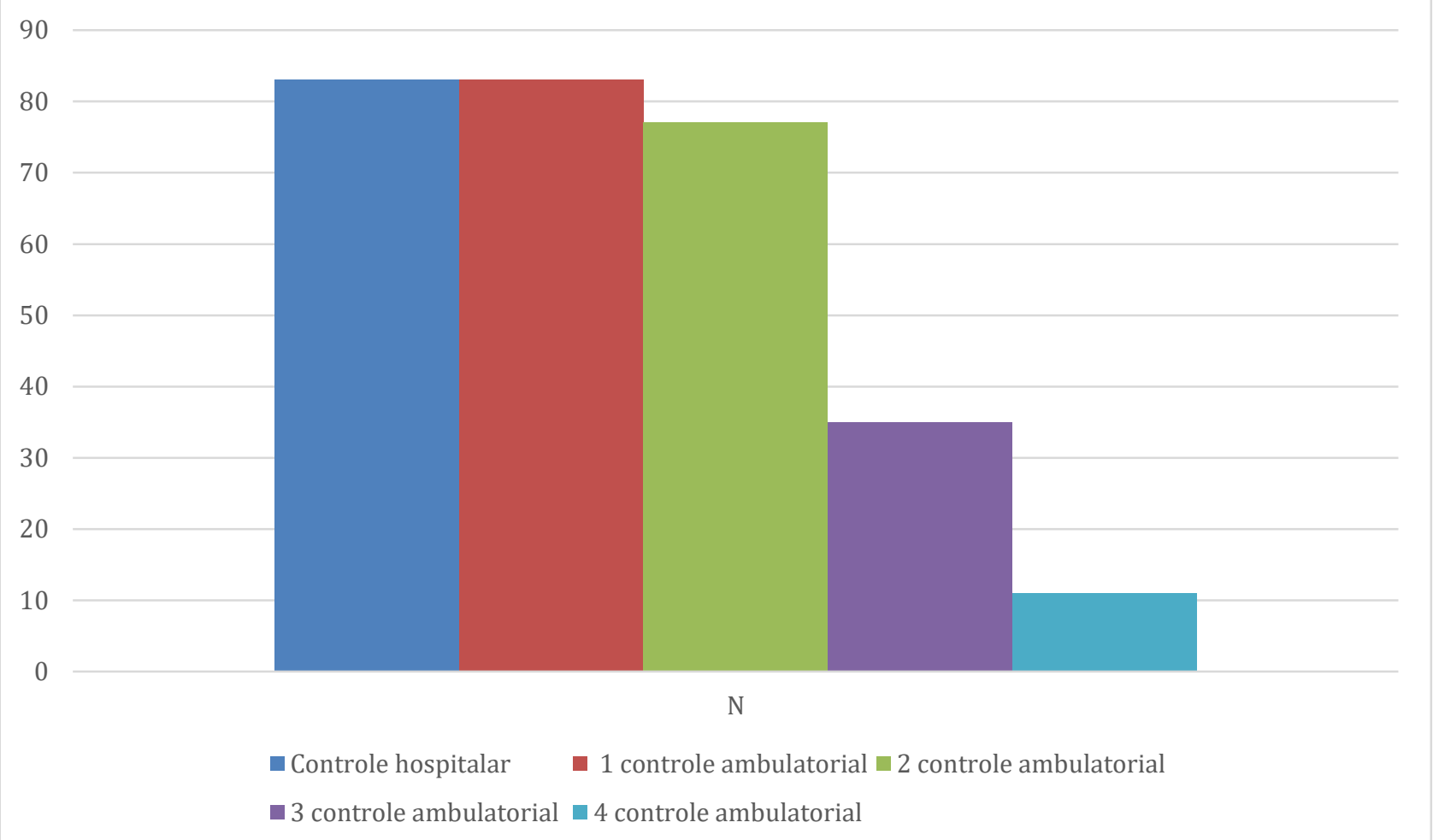

Fonte: Cançado AG, et al, 2020.

Para avaliar o número de lactantes necessários para a avaliação de complicações mamárias, considerando erro alfa de $5 \%$ e beta de $80 \%$, considerando a frequência de amamentação no Brasil nos últimos anos, especialmente na região sudeste, considerou-se a frequência de $50 \%$ de amamentação para puérperas submetidas a parto normal e $30 \%$ para submetidas à cesariana. Considerando grupos paralelos de com 1:1, espera-se 95 pacientes em cada grupo. Entretanto, por ser um estudo inicial em nossos serviços, optou-se por inicialmente realizar um estudo piloto, onde se espera $10 \%$ do total de pacientes calculadas. Todas essas puérperas serão divididas em dois grupos, sendo o grupo A composto por pacientes que evoluíram para parto normal e o grupo $B$ formado por aquelas pacientes que foram submetidas à cesariana. 
Os dados foram digitalizados em formulários eletrônicos de banco de dados Access desenvolvidos especialmente para os estudos. Os resultados obtidos serão expressos como média e desvio padrão para variáveis quantitativas e como frequência absoluta e frequência relativa para as variáveis categóricas. As análises estatísticas foram realizadas com auxílio do software JAMOVI vs 0.8.1.5 para MAC (ios) e utilizando o teste $t$ de Student para comparação de médias entre amostras independentes. Para verificar associação, utilizou-se o teste exato de Fischer quando possível ou o teste do $\mathrm{x} 2$ para variáveis com mais de duas categorias. O nível de significância adotado foi de $5 \%$.

O presente estudo foi aprovado pela Comissão de Ética da Faculdade de Medicina de Barbacena e da instituição coparticipante. Todos os pacientes assinaram o termo de consentimento livre e esclarecido.

\section{RESULTADOS}

Foram estudadas 83 lactantes neste período. A média de idade foi de $30,8 \pm 5,4$ anos e de gestações e partos foi respectivamente $1,8 \pm 1,15$, sendo o valor mínimo de 1 e máximo de seis gestações e $0,95 \pm 1,16$ partos, sendo o mínimo 0 e máximo de cinco partos prévios. Destas pacientes, $64(77 \%)$ foram submetidas a cesariana e $19(22,9 \%)$ evoluíram para parto vaginal. Além disso, $64(77,1 \%)$ tinham realizado pré-natal de alto risco e $19(22,9 \%)$ no serviço de baixo risco das instituições avaliadas. Não houve associação entre o tipo de pré-natal e a via de parto $(p=0,686)$. O motivo para encaminhamento ao alto risco obstétrico foram: doença reumática $(n=2)$, crescimento intrauterino restrito $(n=2)$, hiperplasia adrenal congênita $(n=5)$, diabetes mellitus gestacional $(n=18)$, pré-eclâmpsia $(n=6)$, trombose venosa $(n=2)$, trombofilia $(n=8)$ e outros $(n=39)$. Em outros foram incluídas: doença falciforme, talassemia, diabetes prévio, alterações de volume de líquido amniótico, fator de risco para pré-eclâmpsia, miomatose e idade avançada.

A idade gestacional no parto foi de $37,40 \pm 3,19$ semanas no parto, com média de peso ao nascer de 3169 $\pm 900,87$ gramas. Imediatamente após o parto, durante a internação, $89,2 \%$ das pacientes estavam em lactação exclusiva e receberam alta nessa condição, mas 10,8\% já no hospital estavam em alimentação complementae (Tabela 1). O motivo para a não lactação exclusiva identificados nesse momento foram a hipogalactia, recém-nascidos internados em UTI neonatal e dificuldade de pega, recebendo auxílio do pediatra e do setor de amamentação hospitalar.

Tabela 1 - Frequência da amamentação exclusiva inicial entre as pacientes puérperas e sua associação com a via de parto.

\begin{tabular}{|c|c|c|c|c|c|}
\hline \multirow{2}{*}{$\begin{array}{l}\text { Amamentaçao exclusiva } \\
\mathrm{N} / \%\end{array}$} & \multicolumn{2}{|c|}{ SIM } & \multicolumn{2}{|c|}{ NÃO } & \multirow[t]{2}{*}{ Valor de $p$} \\
\hline & 74 & $89,20 \%$ & 9 & $10,80 \%$ & \\
\hline \multicolumn{6}{|l|}{ Cesariana } \\
\hline $\begin{array}{l}\text { Sim } \\
\text { Não }\end{array}$ & & & & & 0,4 \\
\hline
\end{tabular}

Fonte: Cançado AG, et al, 2020.

Realizou-se associação entre amamentação inicial e tipo de pré-natal $(p=0,43)$, idade, número de gestações $(p=0,18)$, partos $(0,178)$ e tipo de parto, mas também não houve associação estatística $(p=0,4)$ (Tabela 2).

O primeiro controle ambulatorial foi realizado com mediana de 15 dias do parto. Neste período, 55 pacientes $(67,07 \%)$ estavam em amamentação exclusiva, $24(28,92 \%)$ em esquema complementar de amamentação, 3 (3,61\%) em amamentação artificial e 1 (1,60\%) em esquema suplementar (Tabela 3).

Quando questionadas sobre o motivo de mudança de regime de alimentação do recém-nascido a grande maioria respondeu não ter tido leite suficiente (hipogalactia), recém-nascidos ainda internados em UTI neonatal e a dificuldade de pega, apesar dos esforços da equipe (Figura 2). Associou-se amamentação no primeiro controle ambulatorial e número de gestações $(p=0,11)$ e de partos $(p=0,41)$, mas não houve significância estatística.

$\mathrm{Na}$ avaliação do tipo de parto e amamentação incluiu-se todos os estratos de alimentação do recémnascido identificados no período e não houve associação estatística $(p=0,146)$. Para otimizar a avaliação estatística, consideramos a amamentação exclusiva e não exclusiva e sua associação com o tipo de parto. 
Nesse caso, houve associação estatística de forma que o parto cesariano foi fator de risco para a amamentação não exclusiva na mediana de 15 dias de parto. ( $p=0,02 ; O R=3,57 ; \mathrm{IC}=1,11-12,67)($ Tabela 2).

Tabela 2 - Associação entre o tipo de parto e a amamentação exclusiva com 15 dias de parto (mediana).

\begin{tabular}{lccccc}
\hline Amamentação exclusiva & SIM & NÃO & Valor de p & OR (IC) & IC \\
\hline Cesariana & & & & & \\
\hline SIM & 29 & 29 & 0,02 & 3,57 & $1,11-12,67$ \\
Não & 15 & 4 & &
\end{tabular}

Fonte: Cançado AG, et al, 2020.

O segundo controle ambulatorial foi realizado com mediana de 21 dias pós-parto e média de $31,7 \pm 28,1$. Neste período, o aleitamento exclusivo foi de $57,14 \% \quad(n=44)$, artificial de $12,99 \% \quad(n=10)$, complementar $24,68 \%(n=19)$ e a suplementar de 5,19\% $(n=4)$ e 6 pacientes não retornaram para controle. Diagnosticou-se um caso de mastite, um caso de blues puerperal e cinco casos de ingurgitamento mamário.

O terceiro controle foi realizado com 35 pacientes (porque as demais ainda não fizeram esse controle) na mediana de 75 dias do parto e média de $133,00 \pm 153,00$. Neste período, o aleitamento exclusivo foi de $48,57 \%(n=17)$, artificial de $11,43 \%(n=4)$, complementar $37,10 \%(n=13)$ e a suplementar de $2,90 \%(n=1)$.

O último controle foi realizado com mediana de 180 dias e média de $214,00 \pm 167,00$, com apenas quinze pacientes, já que as demais ainda não estão no momento adequado de realizá-lo. Neste período, o aleitamento exclusivo não foi identificado em nenhum bebê, o artificial em $36,36 \%(n=4)$, complementar $54,55 \%(n=6)$ e a suplementar de $9,09 \%(n=1)$. Aqui, a orientação do pediatra desponta como motivadora do aleitamento não exclusivo (Tabela 3). Observa-se ao longo do período, os responsáveis pela mudança de alimentação do recém-nascido foram a hipogalactia (nos primeiros quinze primeiros dias), a agalactia (21 dias) e orientações do pediatra com 75 dias de mediana do parto (Figura 2).

Tabela 3 - Dados sobre as modificações do regime de alimentação do recém-nascido ao longo do acompanhamento (dias - mediana).

\begin{tabular}{cccccccccc}
\hline \multicolumn{2}{c}{ REGIME } & \multicolumn{2}{c}{ EXCLUSIVA } & \multicolumn{2}{c}{ COMPLEMENTAR } & \multicolumn{2}{c}{ SUPLEMENTAR } & \multicolumn{2}{c}{ ARTIFICIAL } \\
\hline 15 dias & $\mathrm{N} / \%$ & 55 & $67,07 \%$ & 24 & $28,92 \%$ & 1 & $1,60 \%$ & 3 & $3,60 \%$ \\
21 dias & $\mathrm{N} / \%$ & 44 & $57,14 \%$ & 19 & $24,68 \%$ & 4 & $5,19 \%$ & 10 & $12,99 \%$ \\
75 dias & $\mathrm{N} / \%$ & 17 & $48,57 \%$ & 13 & $37,10 \%$ & 1 & $2,90 \%$ & 4 & $11,43 \%$ \\
180 dias & $\mathrm{N} / \%$ & 0 & $0,00 \%$ & 6 & $54,55 \%$ & 1 & $9 \%$ & 4 & $36,36 \%$ \\
\hline
\end{tabular}

Fonte: Cançado AG, et al, 2020.

Figura 2 - Identificação dos principais motivadores para a alimentação não exclusiva ao longo do estudo (mediana em dias)

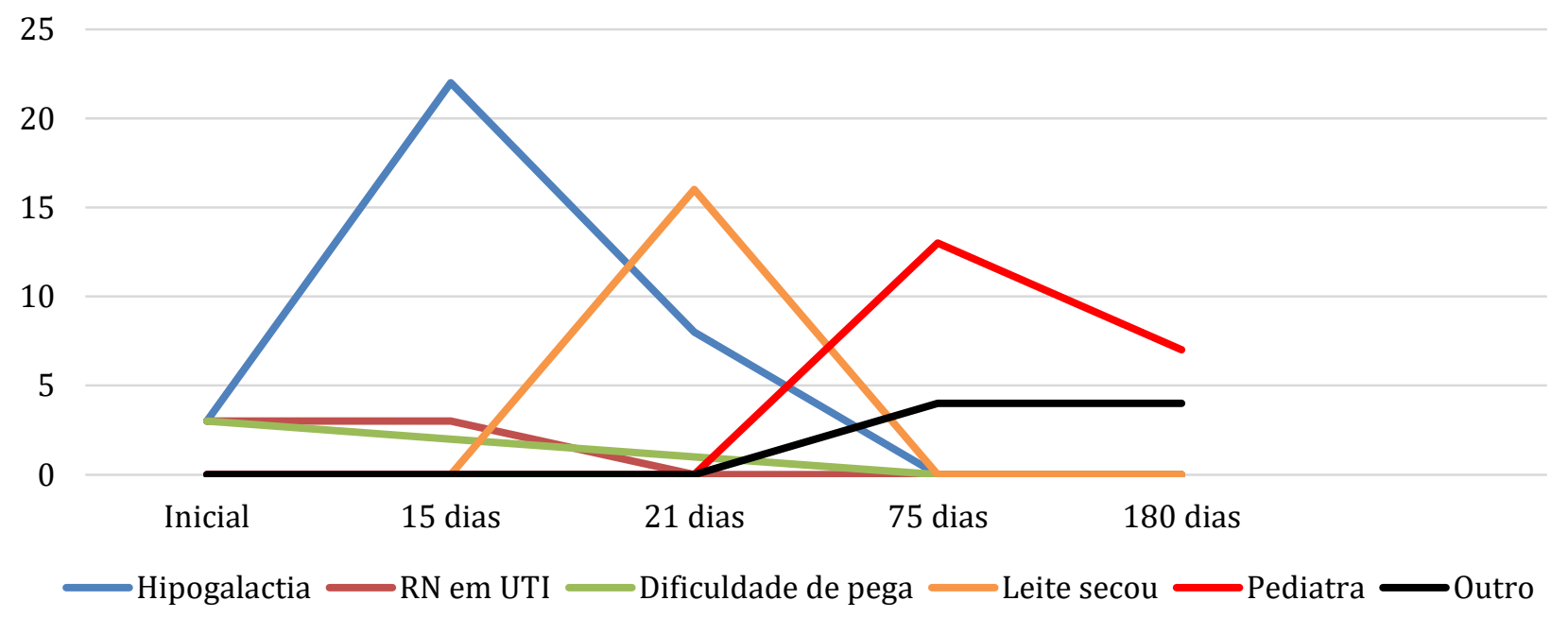

Fonte: Cançado AG, et al, 2020. 


\section{DISCUSSÃO}

A literatura médica é unânime em afirmar que a amamentação exclusiva é superior a qualquer outra forma de alimentar o bebê, mas ainda existem problemas em relação à adesão e o Ministério da Saúde informa que essa prática está abaixo do ideal (BRASIL, 2015). Estudos descritos por Caldeira AP e Goulart EM (2000), evidenciaram menor tempo de aleitamento materno quando os bebês mamaram após as primeiras 6 horas de vida. Bautista LE (1997) descreveu a relação positiva entre início mais rápido da primeira mamada e maior tempo de aleitamento materno. Além disso, já foi notada a associação positiva entre a redução nos índices de desmame e separação do recém-nascido da mãe nas primeiras 6 horas de vida. Imediatamente após o parto, observamos que $89,20 \%$ das pacientes estavam em lactação exclusiva e receberam alta nessa condição, mas $10,8 \%$ já no hospital estavam em alimentação complementar justificado por pouca produção de leite, recém-nascidos internados em UTI neonatal e dificuldade de pega.

Apesar de termos uma expressão importante de lactação exclusiva durante o período de internação, esses $10,8 \%$ nos preocupa, especialmente quando as justificativas estão associadas a pouca produção de leite e dificuldade de pega. O período em que se inicia a produção de leite é chamado de apojadura e acontece em torno de 48 a 72 horas após o parto e o volume de leite produzido tende a aumentar com as mamads. Este conhecimento é importante para a informação do puérpera de que a pequena quantidade de leite produzida nos primeiros dias é normal, não havendo necessidade de complementar com leite ou oferecer outros líquidos. Além disso, os níveis plasmáticos de prolactina elevam-se em resposta à sucção do $\mathrm{RN}$ e estão diretamente relacionados à frequência, duração e intensidade da sucção. Como a prolactina é o hormônio da galactopoiese, a sucção do mamilo pela criança em livre demanda é o elemento chave para o sucesso da amamentação (CHAVES RG, et al., 2007; BRASIL, 2001).

Uma preocupação relevante é que o conjunto de pacientes era composto por gestantes oriundas do alto risco obstétrico (ARO) e a patologia materna poderia ser um fator determinante para o insucesso da lactação exclusiva. Por isso, uma pergunta plausível é se as doenças maternas poderiam influenciar na amamentação e em nossos resultados. Foi identificada associação entre o risco identificado no pré-natal, gestações prévias, partos e idade em relação à amamentação. Por outro lado, Tenório MCDS, et al. (2018) relataram que o hábito tabagista na gravidez [RP $=5,20$; (IC95\% $=1,75-15,33) ; p=0,003]$; intercorrências na gestação $[R P=3,50$; $(\mathrm{IC} 95 \%=1,04-11,77) ; p=0,042]$ e falta de informações sobre aleitamento materno no pré-natal $[\mathrm{RP}=5,44$; $(\mathrm{IC} 95 \%=1,78-16,67) ; p=0,003]$ foram fatores desfavoráveis à amamentação.

Em relação a via de parto, identificou-se que a cesariana é fator de risco para a amamentação exclusiva no puerpério imediato. Segundo Weiderpass E, et al. (1998), hospitais cujo padrão de atendimento pósoperatório dificultem o alojamento conjunto e o aleitamento à livre-demanda, ou ainda permitam a introdução precoce de outros alimentos na dieta infantil, poderiam levar a um maior insucesso do aleitamento. Entretanto, em nossa rotina está o alojamento conjunto e estímulo à sucção ainda na sala de parto. Acreditamos que essa diferença talvez esteja no tipo de cesariana - eletiva ou emergencial - que não foi pesquisada.

À medida que os controles foram realizados, houve aumento das práticas complementares e artificiais de aleitamento. Habitualmente, é esperado que com 180 dias, inicie-se a alimentação artificial da criança e a amamentação deixe de ser exclusiva, mas os dados são graves quando identificamos que com mediana de 75 dias de parto, $49 \%$ das pacientes não mais amamentavam seus bebês. Sabe-se que a oferta de outros líquidos, além do leite materno, é frequente antes dos seis meses, apesar de não ser o indicado pois essa prática pode gerar diminuição no consumo do leite materno e, consequentemente, menor extração e produção do leite, o que contribui para o desmame precoce, menor ganho ponderal da criança, maior risco de ocorrência de diarreias (MARTINS TC, et al, 2019).

Além disso, a complementação precoce ou inadequada também pode trazer prejuízos para a mãe, como o ingurgitamento mamário, mastite, retorno mais rápido da fertilidade, vínculo entre mãe e filho prejudicado e efeitos econômicos (MARTINS TC, et al, 2019). Não se pode esquecer que crianças não amamentadas apresentam maior risco de morrer por diarréia (14X) e por doença respiratória $(3,6 \mathrm{X})$ quando comparadas com crianças amamentadas (VICTORA GG, et al.,1997; VICTORA GG, et al., 1992). Estudo realizado no Brasil, identificou que o risco de hospitalização de uma criança com pneumonia é $17 x$ maior quando não amamentada no primeiro ano de vida e $61 x$ maior, nos primeiros três meses de vida (CESAR JA, et al., 1999). 
Neste estudo não se identificou a prática suplementar no período de internação, porém com o decorrer do estudo houve um aumento na porcentagem desta modalidade até $9 \% \mathrm{com} 180$ dias de parto. Entretanto, estes dados foram melhores que os divulgados por Pinheiro JMF, et al. (2016) que já tinham identificado $16,0 \%$ dessa prática em seu estudo. Quanto aos motivos de indicação, apenas $6,2 \%$ atenderam às recomendações da Iniciativa Hospital Amigo da Criança, destacando-se a hipogalactia como principal motivo $(71,7 \%)$, com maior probabilidade de indicação para os recém-nascidos de parto cesáreo $(p<0,02)$ (PINHEIRO JMF, et al., 2016). Machado MCM, et al. (2014) identificaram que os principais determinantes para o abandono do aleitamento materno são a baixa escolaridade e o trabalho materno, bem como fatores psicossociais, particularmente o apoio do companheiro e a sintomatologia depressiva.

O fato é que a amamentação é negligenciada na prática médica, fazendo supor, especialmente à população leiga, que o leite materno pode ser substituído por produtos artificiais de forma habitual e sem qualquer consequência. Em países de alta renda essa prática é rotineira, pois menos de uma a cada cinco crianças é amamentada aos 12 meses de vida. Mas isso também se repete em países de baixa renda, com menor intensidade, pois a frequência de amamentação é maior que nos países ricos, mas ainda não é a ideal. Relata Victora GG, et al. (2016) que a amamentação é um comportamento positivo de saúde, mais prevalente nos países pobres e que vem contribuindo para a redução das disparidades em saúde entre crianças ricas e pobres. Além disso, mesmo em países de alta renda, esse comportamente altamente positivo poderia reduzir as taxas de morte súbita e de enterocolite necrotizante.

Considerando os objetivos do desenvolvimento sustentável (identificar e acabar com a pobreza, acabar com a fome e assegurar uma vida saudável para todos) percebe-se que a amamentação é capaz de influenciar positivamente. A amamentação permite um melhor desenvolvimento da criança, previne a fome, evita a desnutrição e a obesidade infantil, por garantir os nutrientes necessários e adequados para 0 desenvolvimento do bebê e, por isso, não se pode negligenciar esse efeito positivo no desenvolvimento da criança. Outro aspecto relevante, é o nível intelectual. Estudos mostraram que o leite materno é capaz de influenciar no QI das crianças. Fonseca ALM, et al. (2013) aplicando o teste de Raven em 560 crianças identificaram que a amamentação até o sexto mês de vida promove aumento da capacidade intelectual $(p=0,01)$.

Estudo de Victora GG, et al. (2015) avaliou pessoas aos 30 anos utilizando testes de inteligência (QI) e identificaram que aqueles participantes que foram amamentados por 12 meses ou mais tiveram escores de QI mais altos, com diferença de 3,76 pontos (IC de $95 \%=2,20-5,33$ ), mais anos de educação e maiores rendimentos mensais quando comparados com os que foram amamentados por menos de 1 mês. Desta forma, pode-se dizer que amamentar é importante para vários objetivos, seja para a educação, para a pobreza e até para o crescimento econômico inclusivo. Assim a amamentação pode reduzir o abismo existente entre ricos e pobres e consequentemente, reduzir as desigualdades sociais, que é o décimo objetivo do desenvolvimento sustentável (WHO, 2015; WHO, 2020).

No presente estudo, houve limitações como a falta de retorno de algumas pacientes, com perdas de seguimento ao longo da avaliação. Além disso, ressalta-se que este estudo está sendo continuado, com busca ativa de todas as pacientes, mas este foi apenas o piloto, que teve resultados interessantes para a literatura. Considerando que a hipogalactia, a agalactiae e a conduta do pediatra foram os principais motivadores para a interrupção da amamamentação nesse grupo de pacientes, sugere-se que as pacientes sejam orientadas, especialmente em relação a pega para que a amamentação possa ser realizada de forma sustentada. Outro dado importante, é o comprometimento da equipe de saúde com a amamentação. Os pediatras e profissionais que trabalham diretamente com as puérperas e seus bebês devem dar suporte científico e emocional para que a prática seja mantida.

\section{CONCLUSÃO}

Apesar das pacientes saírem da maternidade amamentando seus bebês, essa prática não se sustenta. A hipogalactia, a agalactia e otientações do pediatra foram os principais motivadores para a suspensão da lactação exclusiva nesse estudo. Além disso, a cesariana foi fator de risco para a não amamentação exclusiva. 


\section{REFERÊNCIAS}

1. BRASIL. Ministério da Saúde. Parto, Aborto e Puerpério. Ministério da Saúde. 2001; 1:1-190.

2. BRASIL. Ministério da Saúde. Secretaria de atençao a saúde. Departamento de Atenção Básica. Saúde da criança: aleitamento materno e alimentação complementar. Cadernos de Atenção Básica à Saúde. 2015. 184p.

3. BAUTISTA LE. Duration of maternal breast-feeding in the Dominican Republic. Rev Panam Salud Publica. 1997; 1:104-11.

4. BOCCOLINI CS. Aleitamento materno: determinantes sociais e repercussões na saúde infantil. Tese. (Doutorado em Saúde Pública). Escola Nacional de Saúde Pública Sergio Arouca. 2012. 196p.

5. CALDEIRA AP, GOULART EM. A situação do aleitamento materno em Montes Claros, Minas Gerais: estudo de uma amostra representativa. J. J Pediatr. 2000; 76. 65072.

6. CÉSAR JA, et al. Impact of breast feeding on admission for pneumonia during postneonatal period in Brazil: nested case-control study. BMJ. 1999; $318: 1316-20$.

7. CHAVES RG, et al. Fatores associados com a duração do aleitamento materno. J Pediatr (Rio J).2007; 83(3(:241-6.

8. DE SIQUEIRA RS, MONTEIRO CA. Amamentação na infância e obesidade na idade escolar em famílias de alto nível socioeconömico. Rev Saude Publica.2007; 41(1): 5-12.

9. ESCUDER MML, et al. Estimativa de impacto da amamentação sobre a mortalidade infantil. Rev Saude Publica. 2003; 37 (3):319-25.

10. FONSECA ALM, et al. Impacto do aleitamento materno no coeficiente de inteligência de crianças de oito anos de idade. J Pediatr. 2013; 89 (4): 346-353.

11. HORTON S et al. Breastfeeding promotion and priority setting in health. Heal Police Plan.1996; 11 (2): $156-68$.

12. JELLIFFE DB. Commerciogenic Malnutrition. Nutr Rev. 1972; 30 (9):199-205.

13. MACHADO MCM, et al. Determinants of the exclusive breastfeeding abandonment: Psychosocial factors. Rev Saude Publica. 2014; 48 (6): 985-94.

14. MARTINS TC, et al. Fatores associados ao tempo de aleitamento materno exclusivo e total em creches municipais de Juiz de Fora-MG, BRASIL. DEMETRA. Aliment Nutr Saúde. 2019; 26 (14): e43583.

15. MCVEA KL, et al. The role of breastfeeding in sudden infant death syndrome. J Hum Lct. 2000; 16 (1): $13-20$.

16. OWEN CG, et al. Effect of infant feeding on the risk of obesity across life course: a quantitative review of published evidence. Pediatrics. 2005; 115: 1367-77.

17. PINHEIRO JMF, et al. Prevalência e fatores associados à prescrição/solicitação de suplementação alimentar em recém-nascidos. Rev Nutr. 2016; 18 (29): 367-75.

18. ROTH DE, et al. Acute lower respiratory infections in childhood: opportunities for reducing the global burden through nutritional interventions. Bulletim World Organ. 2008;86:356-64.

19. TENÓRIO MCDS, et al. Factors associated with the lack of breastfeeding upon discharge from hospital in a public maternity facility in maceió, alagoas, Brazil. Cienc e Saude Coletiva. 2018; 1 (11): 3547-56.

20. VICTORA CG, et al. Amamentação no século 21: epidemiologia, mecanismos, e efeitos ao longo da vida. Epidemiol. Serv. Saúde. 2016:1-24.

21. VICTORA CG, et al. Breast-feeding, nutritional status, and other prognostic factors for dehydration among young children with diarrhoea in Brazil. Bull World Heal Organ. 1992; 7 (1): 467-75.

22. VICTORA CG, et al. Evidence for protection by breast-feeding against infant deaths from infectious diseases in Brazil. Lancet. 1987; 2 (1): 319-22.

23. VICTORA CG, et al. Association between breastfeeding and intelligence, educational attainment, and income at 30 years of age. A prospective birth cohort study from Brazil. The Lancet. 2015; 3(4): E199-E205.

24. WEIDERPASS E, et al. Incidence and duration of breast-feeding by pattern of delivery: A longitudinal study in Southeastern Brazil. Rev Saude Publica. 1998; 32(3): 225-31.

25. WHO. WORLD HEALT ORGANIZATION. Agenda 2030. ONU. Transformando Nosso Mundo: A Agenda 2030 para o Desenvolvimento Sustentável. 2015

26. WHO. WORLD HEALT ORGANIZATION. Duration of exclusive breastfeeding: conclusions and recommendations 54th World Health Assembly. Geneva 2001. UNICEF Glob Strateg Infant Young Child Feed. 2001. 